\section{Revista Brasileira de Administração Científica}

Brazilian Journal of Scientific Administration

Abr a Jun 2020 - v.11 - n.2

\title{
Mentoria formal como estratégia de ação para a formação de competências criativas
}

Este ensaio teórico tem o objetivo de apresentar o processo de mentoria formal, dentro da lógica das funções de mentoria, como uma alternativa estratégica interessante para a formação de classes criativas em oposição à visão tradicional de captação de recursos humanos criativos. O que se percebe é que parte dos estudiosos tem se concentrado na atração de pessoas criativas e num certo determinismo histórico-geográfico que imana valores culturais os quais por sua vez geram, respectivamente, pessoas criativas, classes criativas e, consequentemente, contextos criativos que, a partir daí se retroalimentariam. Essa lógica é claramente suportada pela abordagem estratégica baseada em recursos, dentro de uma perspectiva cultural, onde pessoas, sua cultura e sua criatividade são elementos valiosos, raros e de difícil imitação. Resultados de pesquisas têm comprovado a validade da mentoria como um mecanismo crítico para o desenvolvimento de talentos em grandes empresas, especialmente no setor público. Como nem sempre é possível, pelo menos em um instante inicial, atrair recursos valiosos em quantidade significante, verifica-se que a formação de tais recursos humanos possa significar uma vantagem competitiva interessante às cidades que têm a pretensão de se destacar competitivamente por tal capacidade de inovação, criatividade e geração de novas perspectivas econômicas, sociais, políticas e, sobretudo, culturais.

Palavras-chave: Mentoria; Estratégia; Cultura; Criatividade; Visão Baseada em Recursos.

\section{Formal mentoring as an action strategy for the formation of creative skills}

This theoretical essay aims to present the formal mentoring process, within the logic of mentoring functions, as an interesting strategic alternative for the formation of creative classes as opposed to the traditional vision of capturing creative human resources. What is perceived is that part of the scholars has concentrated on the attraction of creative people and on a certain historical-geographical determinism that emanates cultural values which in turn generate, respectively, creative people, creative classes and, consequently, creative contexts that, from there, they would feed back. This logic is clearly supported by the strategic approach based on resources, within a cultural perspective, where people, their culture and their creativity are valuable, rare and difficult to imitate. Research results have proven the validity of mentoring as a critical mechanism for talent development in large companies, especially in the public sector. As it is not always possible, at least in an initial moment, to attract valuable resources in a significant amount, it appears that the formation of such human resources can mean an interesting competitive advantage to cities that intend to stand out competitively for such capacity of innovation, creativity and generation of new economic, social, political and, above all, cultural perspectives.

Keywords: Mentoring; Strategy; Culture; Creativity; Resource Based View.

Topic: Comportamento Organizacional

Reviewed anonymously in the process of blind peer
Received: $\mathbf{2 1 / 0 3 / 2 0 2 0}$

Approved: 02/04/2020
Marcela Rebecca Pereira (iD

Universidade Federal de Pernambuco, Brasil

http://lattes.cnpq.br/6034842332008434

http://orcid.org/0000-0002-4989-3941

marcelarebecca@hotmail.com

José Ricardo Costa de Mendonça (iD)

Universidade Federal de Pernambuco, Brasil

http://lattes.cnpq.br/5603289094229665

http://orcid.org/0000-0001-7718-984X

ircm@ufpe.br

Luiz Sebastião dos Santos Júnior (iD)

Universidade Federal de Pernambuco, Brasil

http://lattes.cnpq.br/6229547391911379

http://orcid.org/0000-0002-9172-6688

luizssjr@hotmail.com 


\section{INTRODUÇÃO}

Para Carlos (2013), a forma como a sociedade se auto-(re)produz, apesar de acontecer em um espaço geográfico específico, acontece também em um espaço histórico, igualmente específico, associado à dimensão temporal e em escalas diferenciadas. Serpa (2013) reforça esse pensamento ao defender que para além da realidade natural, nós, enquanto sociedade, construímos nossa própria realidade social. Esse contexto geográfico-histórico acaba por imanar a criação de contextos mais específicos, dentre os quais, para este trabalho, de destacam os contextos ditos como criativos, onde se desenvolvem indústrias, empresas e clusters relacionados à criatividade (O'CONNOR et al., 2010).

Para atender a demanda de tais contextos criativos ou mesmo para gerar e manter tais contextos, Florida (2003) defende que é necessária a existência de uma "classe criativa". Contudo, Vinodrai (2013) expõe as dificuldades econômico-político-ideológicas em criar sistemas de inovação que atraiam as pessoas criativas para preencher esse espaço de desenvolvimento em um momento histórico em que a oferta de empregos criativos cresce proporcionalmente mais do que a dos empregos tradicionais.

Amabile (1996) parte da lógica de que é preciso destacar os antecedentes geradores de criatividade. Tais antecedentes dizem respeito ao domínio de habilidades relevantes, processos mentais, motivação para a tarefa e contexto social. Erez et al. (2010), a partir desta lógica, propõem um modelo conceitual que destaca a importância dos contextos culturais no processo de geração de pessoas criativas.

O que se percebe é que parte dos estudiosos tem se concentrado na atração de pessoas criativas e num certo determinismo histórico-geográfico que imana valores culturais que por sua vez geram, respectivamente, pessoas criativas, classes criativas e contextos criativos que, a partir daí se retroalimentariam.

A teoria baseada em recursos (WERNERFELT, 1984) prevê que a liderança estratégica depende da base de recursos da firma, contudo, claramente a visão não se adéqua apenas às firmas, mas a diversos contextos organizacionais. Um aspecto essencial da teoria, realçada por Mintzberg et al. (2010) ao enquadrála dentro da metáfora da cultura é a de que os recursos podem ser desenvolvidos e o recurso mais difícil de ser imitado deriva de questões culturais e isso é próprio dos recursos humanos. Em resumo, desenvolver recursos humanos é talvez uma das principais vantagens competitivas organizacionais, pois se configura como valioso, raro, de difícil imitação, desde que devidamente explorado pela organização (BARNEY, 1991).

A proposta desse ensaio tem caráter mais intervencionista e parte do princípio de que é possível, conjuntamente com a estratégia de atração de pessoas criativas, instaurar estratégias de formação de uma classe criativa a partir das pessoas que já se encontram em um determinado espaço histórico-geográfico e que, precisam inclusive, ser incluídas para não ficarem à margem do processo de desenvolvimento social. Este ensaio pretende apresentar, portanto, a mentoria formal como uma dessas estratégias de formação de classe criativa. A adoção de mentores formais dentro de um correto direcionamento das funções e níveis de mentoria (KRAM, 1988; ZEY, 1984) como variáveis moderadoras complementares ao modelo proposto por Erez et al. (2010) é, portanto, o objetivo principal deste ensaio. 


\section{METODOLOGIA}

Eriz et al. (2010) propõem um modelo conceitual (Figura 1) para compreensão de como os valores culturais acabam por interferir, moderadas por variáveis de contexto social e da tarefa no tipo de criatividade que é gerada, ser voltada para a originalidade ou para a utilidade.

Segundo as proposições desses autores, valores culturais que privilegiam o individualismo, a menor distância de poder, e uma maior tolerância à incerteza, moderadas por contextos de maior privacidade, menor supervisão e tarefas menos rígidas tenderiam a gerar indivíduos mais criativos do ponto de vista da originalidade. Por sua vez, culturas mais coletivistas, de poder mais centralizado e distante, com menor tolerância ao risco, moderadas por trabalhos em grupo, com maior supervisão e tarefas mais rotinizadas, tenderiam a gerar indivíduos mais criativos de uma forma mais utilitarista.

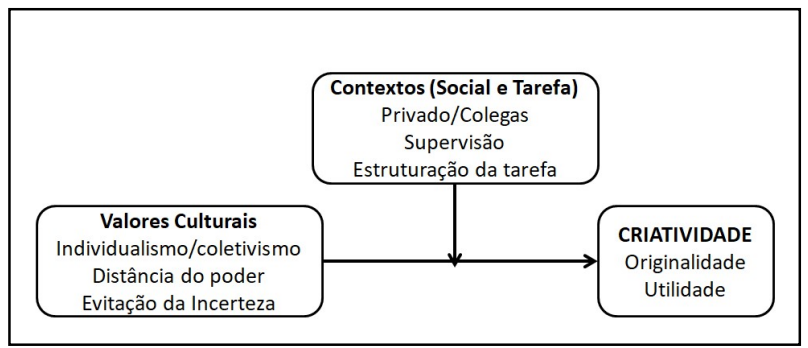

Figura 1: Modelo de Eriz et al. (2010).

O Modelo de Eriz et al. (2010) apesar de ser bem elucidativo quanto à lógica existente entre os elementos que geram criatividade, ainda funciona sob um caráter mais determinístico-conceitual, que permite, segundo os próprios autores, gerar novas ideias e estudos mais aprofundados quanto ao caráter dinâmico de tais elementos. Assim, nessa perspectiva, pretende-se aqui defender que a mentoria formal, com suas funções psicossociais e de carreira, podem agir como estratégias de ação para o desenvolvimento de profissionais criativos.

A segunda hipótese da tese proposta por Santos Júnior (2017) em seu estudo sobre liderança servidora e mentoria é a de que os níveis de comprometimento do mentor determinam o grau de amplitude de cada uma das funções de mentoria exercidas para com o mentorado, tal como é ilustrada pela figura 2.

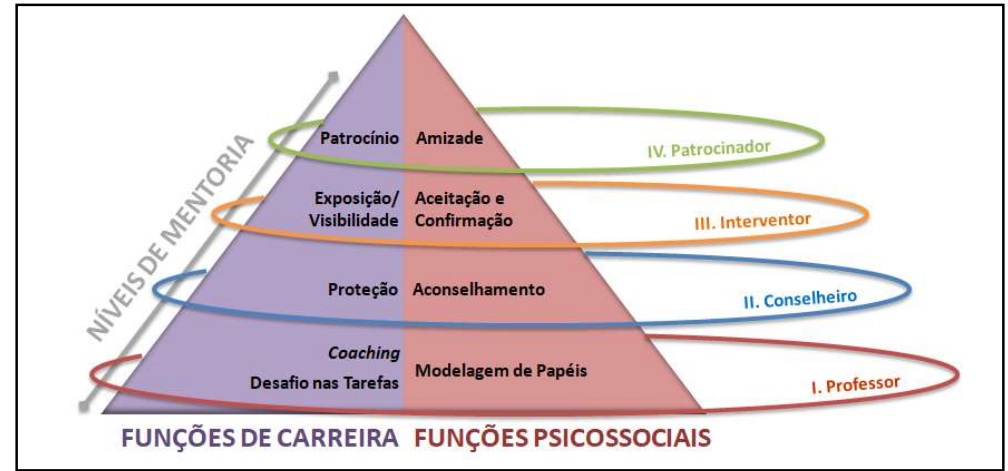

Figura 2: Relação entre níveis e funções de mentoria, adaptado de Santos Júnior (2017).

Aliando-se as duas proposições, tanto a de Eriz et al. (2010) como a de Santos Júnior (2017), é possível identificar novas implicações teóricas a serem testadas (Figura 3). Esse estudo pretende argumentar que a 
variação de elementos culturais pede funções de mentoria específicas para que sejam gerados os dois tipos de criatividade.

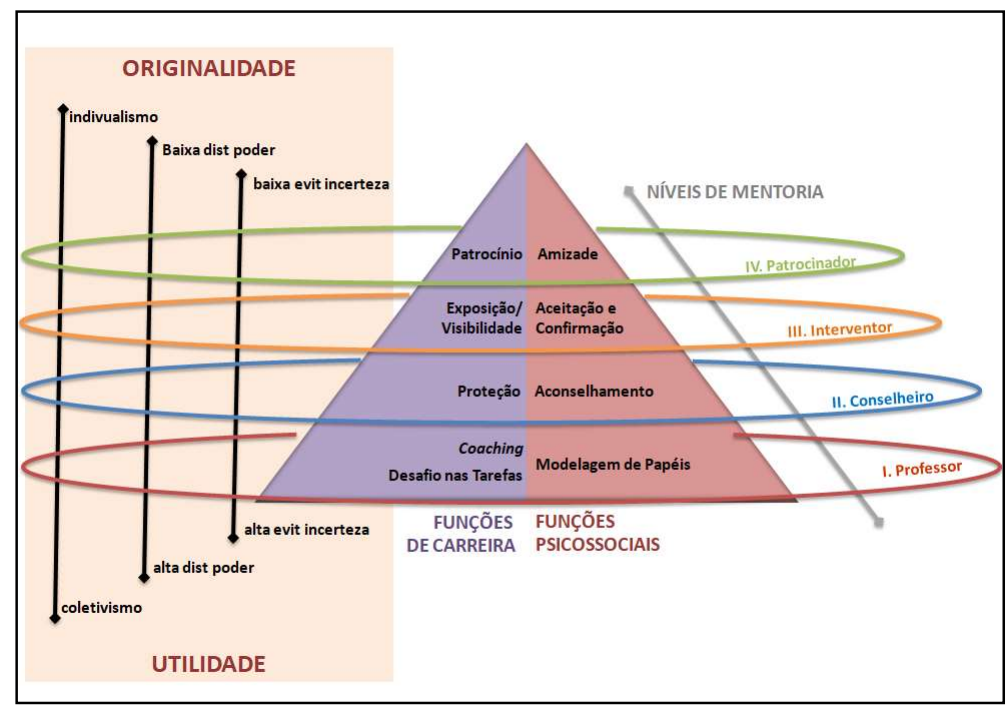

Figura 3: Modelo de estratégias de ação para a formação de classes criativas a partir da mentoria formal.

Assim, para culturas de maior individualismo, menor distância do poder e maior tolerância à incerteza, as funções de amizade e de patrocínio seriam preponderantes. Ambientes culturalmente mais coletivistas, de maior distanciamento do poder e de menor ambiguidade de papeis, tenderia a pedir por uma mentoria baseada mais nas funções de modelo, de coaching e de estabelecimento de tarefas desafiadoras.

Situações intermediárias mais voltadas para a originalidade, tenderiam a buscar funções de mentoria mais ligadas à exposição/visibilidade e de aceitação e confirmação. Situações intermediárias mais voltadas para a criatividade do tipo utilitária, pediriam por funções de mentoria mais ligadas à proteção e ao aconselhamento.

\section{DISCUSSÃO TEÓRICA}

É notório que por seu caráter retransmissor de conhecimentos, o fenômeno da mentoria está associado à própria história da humanidade. A origem da palavra data de 800 a.C. onde n'A Odisseia, de Homero, sua personagem principal, Ulisses, tem na figura do idoso Mentor, um amigo e conselheiro, que desempenha ainda o papel de tutor de seu filho Telêmaco. Neste épico, várias vezes Atena, a deusa da sabedoria, assume a forma de Mentor para aconselhar Ulisses e seu filho (ENSHER et al., 1997).

Embora esses fatos demonstrem que a mentoria não se trata de algo inédito no campo do conhecimento humano, somente a partir da década de 70 do século passado que estudos sistemáticos têm sido conduzidos em maior profundidade (CHAO, 1997), produzindo-se assim um relativo arcabouço teórico sobre questões associadas ao tema.

Santos Júnior (2005) argumenta que é razoável afirmar que a compreensão do fenômeno da mentoria esteja relacionada a diferentes estilos de vida que diferentes grupos de pessoas possuem, de modo que, ao menos inicialmente, se faz necessário conhecer percepções particulares da mentoria, especialmente no tocante às suas fases, níveis e funções. 


\section{Mentoria informal e mentoria formal}

Dentre os diversos temas associados à mentoria, a questão de sua formalização vem recebendo uma crescente atenção. A origem dessa tendência provavelmente se encontra nos resultados de pesquisa obtidos por Dirsmith et al. (1985) que comprovaram a validade da mentoria como mecanismo crítico para o desenvolvimento de talentos em grandes empresas públicas americanas. Para Viator (1999) desde então, as empresas têm investido mais agressivamente no desenvolvimento de programas formais de mentoria.

Kram (1985) e Ragins et al. (2000) trabalharam para determinar a contextualização dessas denominações. Segundo estes, na mentoria informal o desenvolvimento do relacionamento se dá por ajustes mútuos entre mentor e protegido, sendo, portanto, a forma natural como o fenômeno da mentoria tenha se disseminado ao longo da história. Por sua vez, a mentoria formal tem seus relacionamentos muitas vezes determinados por uma terceira parte.

Com relação ao processo motivacional, a mentoria informal conta com aspectos intrínsecos mais fortes, devido ao processo de escolha já citado e às questões de comportamento que evidenciam companheirismo. Já a mentoria formal, tem sua motivação baseada em aspectos mais extrínsecos como remuneração, status e progressão de carreira (KRAM, 1985).

Quanto ao tempo de duração de uma mentoria, do ponto de vista informal não existe uma estrutura pré-definida. Sob a ótica formal, é necessária toda uma estrutura planejada, que preveja inclusive as principais fases de um relacionamento de mentoria, que segundo Kram (1983) são: iniciação, amadurecimento, separação e redefinição.

Quanto ao propósito, os relacionamentos informais de mentoria estão mais voltados para a consecução dos objetivos de longo prazo dos mentorados. Já os programas formais buscam a consecução de objetivos de curto prazo, voltados para orientação do novo empregado em uma espécie de treinamento onthe-job (KRAM, 1985).

Assim sendo, vê-se que o modelo informal oferece mais condições de sucesso em relacionamentos de mentoria e é muito comum em quaisquer tipos de organização. A dificuldade consiste em reproduzir as vantagens do modelo informal sob uma perspectiva formal de modo que o desenvolvimento de relações de mentoria possa favorecer o desenvolvimento dos recursos humanos, especialmente os voltados à construção da criatividade (RAGINS et al., 2000; ALLEN et al., 1997). Metaforicamente, seria como a "reprodução em cativeiro" de animais habituados a uma vida selvagem.

Embora admita as vantagens trazidas para a organização quanto aos relacionamentos formais de mentoria, Feldman (1999), chama a atenção para o fato de que muitos jovens mentorados têm tido suas carreiras profissionais obstruídas por disfunções no relacionamento com seus mentores. Contudo, o mesmo autor argumenta que as razões do fracasso da dinâmica interpessoal desses relacionamentos de mentoria estão em não raras vezes associadas tanto ao mentor quanto ao mentorado.

Estudos têm identificado que a adoção ou não de programas de mentoria não é uma simples questão de "ou tudo ou nada", mas não se pode negar que em alguns casos, indivíduos que não foram submetidos a 
um relacionamento de mentoria expressam atitudes mais positivas do que aqueles que participaram de relacionamentos malconduzidos. Tais estudos concluem ainda que a mentoria de boa qualidade traz as vantagens esperadas do programa, porém enfatiza que relacionamentos de mentoria inadequados podem vir a ser mais prejudiciais do que não adotar nenhum modelo formal de mentoria (RAGINS et al., 2000).

Apesar das ressalvas aqui feitas quanto à formalização dos relacionamentos de mentoria, negligenciar tais intervenções a priori seria limitar a força do construto e os benefícios que têm sido registrados na literatura desenvolvida sobre o assunto (ZEY, 1984; TYLER, 1998),

\section{Níveis de mentoria}

Zey (1984) enfatiza que o relacionamento entre mentores e protegidos pode ser descrito em termos de uma hierarquia quanto à complexidade da atividade de mentoria e propõe quatro níveis característicos, onde o investimento do mentor no mentorado aumenta gradativamente:

No primeiro nível, o de ensino, o protegido recebe de seu mentor instruções sobre a realização do trabalho, peculiaridades da função, modos de comportamento, entre outros. Nesse nível, o principal investimento do mentor diz respeito ao tempo gasto para a transmissão desses conhecimentos. No nível seguinte, o mentor oferece apoio pessoal e aconselhamento psicológico, buscando melhorar a autoestima do mentorado. Seu comprometimento nesse nível é prioritariamente emocional. Na etapa posterior, terceiro nível, a atividade do mentor passa a ser de interventor junto à organização em relação às necessidades de seu protegido. O mentor investe nessa fase, principalmente em sua reputação e em seus relacionamentos dentro da organização. No nível mais elevado o mentor coloca em evidência além da sua reputação a sua própria carreira, pois nessa etapa o mentor assume responsabilidade pelo protegido, recomendando-o, por exemplo, para uma promoção.

É interessante observar que o modelo de hierarquia de comprometimento do mentor proposto por Zey (1984) relaciona de certo modo a escalada nessa hierarquia à maturidade dos relacionamentos de mentoria. Consequentemente, para o autor, as dificuldades na implementação de modelos formais de mentoria, podem estar associadas aos altos graus de envolvimento e de investimento pessoal a que se propõe o mentor com o amadurecimento de suas relações com seu protegido e, consequentemente, ao alto custo que pode vir a ter ao tomar tais atitudes. Em outras palavras, como a mentoria informal costuma ocorrer de modo natural é, portanto, também natural que o mentor aceite assumir nesse modelo, um maior envolvimento com o mentorado do que em uma simples designação formal por parte da organização da sua atividade como mentor.

\section{Funções de mentoria}

Estudos realizados acerca das funções desempenhadas pelos mentores, classificam-nas em dois grupos distintos: funções psicossociais (Quadro 1) e funções de carreira (Quadro 2). Tais funções foram desenhadas a partir de pesquisas qualitativas realizadas por $\operatorname{Kram}(1983,1985)$ e $\operatorname{Kram}$ et al. $(1985)$ e posteriormente sistematizadas e validadas em instrumento de coleta de dados por Noe (1988). 
Quadro 1: Funções psicossociais da mentoria.

MODELO
A função psicossocial onde o mentor serve de modelo para o
das funções mais observadas nas pesquisas de Kram (1988) e
Noe (1988). Nesta função o mentor proporciona ao
mentorado uma 'inspiração' para a sua formação, a qual está
baseada nos valores, nas atitudes e nas ações do mentor

\section{ACONSELHAMENTO}

A função de aconselhamento torna o mentor uma espécie de depositário dos medos, dúvidas e angústias do mentorado. Esta função possibilita ao mentor conhecer os principais problemas que podem representar entraves à progressão do mentorado. É, portanto, uma das mais importantes atribuições do mentor e está relacionada inclusive a etimologia do próprio fenômeno, haja vista que era exatamente a função de conselheiro que o personagem épico de Mentor exercia quando incorporado pela deusa da sabedoria Atena. (HEGSTAD; 1999; KRAM, 1988).

AMIZADE

A função de amizade diz respeito a uma interação social do mentor com seu mentorado dentro do ambiente de trabalho, mas sobretudo fora dele. Acredita-se que em um ambiente mais informal, baseado em amizade sincera, há uma melhoraria significativa da relação de mentoria, tornando as partes assim mais próximas e satisfeitas com o relacionamento. (NOE, 1988; KRAM, 1985).

As funções de mentoria ditas psicossociais, ou seja, que servem de suporte ao desenvolvimento social e psicológico do mentorado são, segundo Kram (1985): amizade; aconselhamento; aceitação e confirmação; e modelo (Figura 1). Já as funções de carreira englobam: exposição/visibilidade; coaching; proteção; patrocínio; e desafio nas tarefas (Figura 2). As funções de carreira estão associadas a fatores organizacionais e servem de suporte para o crescimento profissional do mentorado.

Figura 2: Funções de carreira na mentoria.

COACHING
Para Kram (1988), dentre as funções de carreira, a função de
coaching é a que está mais relacionada às tarefas a serem
desempenhadas pelo mentorado. O mentor exerce um papel
semelhante ao de um treinador de esportes, chamando a
atenção do mentorado para seus pontos fortes e fracos, suas
oportunidades de melhoria e as ameaças a sua posição. Esta
retroação é vital para o aprendizado do mentorado que
entende pouco inicialmente dos aspectos mais particulares
(burocráticos) de sua função.

\section{PROTEÇÃO}

A proteção, entretanto, surge como uma função que o mentor desempenha quando a exposição do mentor pode ser considerada prematura. A importância dessa função reside no fato de que o mentor possui a experiência necessária para distinguir os tipos de críticas a que o mentorado pode ser submetido. Em determinado momento o jovem aprendiz pode ser prejudicado se for exposto desnecessariamente, prejudicando assim o seu desenvolvimento pessoal. (KRAM, 1985).

\section{PATROCÍNIO}

A função de patrocínio, ou seja, de apoio explícito do mentor ao mentorado é, segundo Kram (1988) a que mais foi observada em seu estudo. Entende-se que tal função está intrinsecamente relacionada à própria essência da mentoria. A principal finalidade dessa função é ajudar o mentorado a se desenvolver em uma organização e/ou profissão. A função representa porém um risco para a reputação do mentor quando da não correspondência do mentorado às expectativas nele depositadas e um risco para o mentorado caso o mentor venha a se afastar por quaisquer motivos.

\section{CONCLUSÕES}

É importante pensar questões como cultura e criatividade dentro da visão processual descrita por Van de Ven et al. (1995), onde há início, desenvolvimento, maturidade e declínio, o mesmo acontecendo com

\section{DESAFIO NAS TAREFAS}

A função de promover tarefas com desafios é para Kram (1985) vital ao crescimento profissional do mentorado. O mentor deve ter a capacidade de oferecer ao mentorado tarefas desafiadoras para cada estágio específico de seu crescimento profissional, mesmo porque, desafios considerados instransponíveis para a situação atual do mentorado podem causar mais prejuízos à formação do mentor do que mesmo vantagens. O desafio moderado, em conjunto com o devido suporte proporcionado

A função de exposição/visibilidade diz respeito ao empenho voluntário do mentor para a criação e a delegação de oportunidades ao mentorado, direcionando-o para assumir responsabilidades e para desempenhar tarefas que o ponham em organização ou da profissão. (KRAM, 1985). 
a mentoria, especialmente a mentoria formal. Muzzio et al. (2012) defendem um modelo de avaliação cultural de cunho interpretativista onde a contexto regional deriva um contexto de cultura organizacional que privilegia as interações organizações em múltiplos níveis. Segundo eles, nesse modelo prevê-se que adaptações mercadológicas e de relacionamentos pessoais podem ser favorecidos por contextos regionais.

Assim, acredita-se que aspectos da cultura como a criatividade nas organizações e o surgimento de classes criativas podem ser geradas a partir de contextos mais particulares. Nessa lógica, como a mentoria também pode ser vista como um processo de desenvolvimento de recursos humanos (ZEY, 1984), e como um mentor é conhecedor da cultura local, a transmissão de conhecimentos se torna mais direta e facilitada.

Desta forma, ao se desenvolver sistemas de inovação regionais (BIALK-WOLF et al., 2013), à luz do que foi apresentado, parece ser interessante inserir às perspectivas teóricas que sustentam o delineamento de tais programas, a figura de um mentor, mais especificamente um mentor formal e de forma mais sistemática a inclusão de um programa formal gerador, mantenedor e desenvolvimentista de mentoria, especialmente voltada aos setores que compõe as classes criativas definidas por Florida (2003), porém não apenas estes setores haja visto que variações culturais podem determinar para alguns espaços setores criativos particulares, idiossincráticos e fundamentais à região. Artesãos com pouco ensino formal (ou mesmo analfabetos) podem ser mais necessários ao desenvolvimento criativo de uma dada região em um dado momento histórico-político de sua evolução do que categorias profissionais formadas em ensino superior.

As proposições aqui levantadas nesse trabalho precisam ser verificadas em situações empíricas, o que é natural em se tratando de um ensaio teórico e as evidências apresentadas no decorrer do texto mostram que existe base teórica que suporte à geração desse modelo mais dinâmico que traz à literatura de Cultura, Criatividade e Espaço uma ferramenta prática que pode tornar possível o desenvolvimento de talentos criativos sem que haja uma exclusiva dependência para com propostas de atração de recursos humanos criativos advindos de outras regiões. Tal ferramenta seria a adoção de programas formais de mentoria voltadas para suportar classes criativas.

Inclusive é possível pensar em uma função específica para a atração de talentos para uma região, que é o de cumprir uma missão de desenvolver novos talentos, permitir o crescimento e evolução de novos territórios a partir de uma renovação criativa, dentro da lógica defendida pelos teóricos das trocas sociais (KRAM, 1988), onde $B$ pode repassar para $C$ aquilo que recebeu de $A$, e que não pode devolver a $A$. Assim se perpetua um sentimento de gratidão que pode ser apoiado dentro de uma lógica que está muito próxima dos conceitos de Liderança Servidora (SANTOS JÚNIOR, 2017).

Percebe-se, portanto, que este estudo permite assim gerar insights dentro dessa lógica formativa que parece pouco estudada (e praticada) na formação de cidades criativas. Além disso, o link com a abordagem teórica desenvolvida para a Mentoria, que tem sua particular robustez, pode tornar ainda mais rica as contribuições teóricas acerca dos temas associadas à cultura, ao espaço e a criatividade. Um outro aspecto que parece emergir deste trabalho é que a gestão da criatividade pode estar associada a modelos mais contemporâneos de liderança tal como a liderança servidora e a enfoque sociais como a teoria das 
trocas sociais.

Por fim, acredita-se que a inserção tanto da base teórica de mentoria quanto das experiências práticas de intervenção de mentoria formal geram valor dentro da lógica de formação de classe criativas. Obviamente não se afirmar aqui que esta seria a única solução, mas uma das soluções e que, a priori, parece ser bem concreta, útil e passível de aplicação sem maiores prejuízos. Experimentos de implementação de programas de mentoria formal que vêm sendo aplicadas em alguns contextos criativos, como os do CESAR (Centro de Estudos de Sistemas Avançados do Recife) em Pernambuco, que está utilizando mentores para treinar seus incubados (SALGUES, 2004; CESAR, 2018), pode significar uma oportunidade ímpar para o levantamento de evidências empíricas quanto ao modelo aqui proposto.

\section{REFERÊNCIAS}

ALLEN, T. D.; RUSSELL, J. E. A.; MAETZKE, S. B.. Formal peer mentoring: factors related to protégés' satisfaction and willingness to mentor others. Group \& Organization Management, v.22, n.4, p.488-507, 1997.

AMABILE, T. M.. Creativity in context. New York: Westview, 1996.

BARNEY, J.. Firm resources and sustained competitive advantage. Journal of Management, v.17, n.1, p.99-120, 1991.

BIALK-WOLF, A.; PECHLANER, H.; NORDHORN, C.. The role of culture in building regional innovation systems and its impact on business tourism - The case of the Nuremberg Metropolitan Region. Poznań University of Economics Review, v.13, n.4, p.111-129, 2013.

CARLOS, A. F. A.. Da 'Organização' à 'Produção' do Espaço no Movimento do Pensamento Geográfico. In: CARLOS, A. F. A.; SOUZA, M. L.; SPOSITO, M. E. B.. A Produção do Espaço Urbano: Agentes e Processos, Escalas e Desafios. São Paulo: Contexto. 2013. p.53-73.

CESAR. Centro de Estudos de Sistemas Avançados do Recife. Consultoria ou Mentoria?: Como desenvolvemos os empreendimentos do CESAR.LABS através dessas abordagens: Parte 2. CESAR, 2018.

CHAO, G. T.. Mentoring phases and outcomes. Journal of Vocational Behavior, v.51, p.15-28, 1997.

DIRSMITH, M. W.; COVALESKI, M. A.. Informal communications, nonformal communications and mentoring in public accounting firms. Accounting, Organizations and Society, v.10, n.2, p. 149-169, 1985.

ENSHER, E. A.; MURPHY, S. E.. Effects of race, gender, perceived similanty, and contact on mentor relationships. Journal of Vocational Behavior, v.50, n.3, p.460-481, 1997.

EREZ, M.; NOURI, R.. Creativity: The Influence of Cultural, Social, and Work Contexts. Management and Organization Review, v.6, n.3, p.351-370, 2010.
FELDMAN, D. C.. Toxic mentors or toxic protégés? A critical re-examination of dysfunctional mentoring. Human Resource Management Review, v.6, n.3, p.247-278, 1999.

FLORIDA, R.. Cities and the Creative Class. City and Community, v.1, n.2, p.3-19, 2003.

KRAM, K. E.. Mentoring at work: developmental relationships in organizational life. Glenview: Scott, Foresman, 1985.

KRAM, K. E.. Mentoring at work. Lanham: University Press of America, 1988.

MINTZBERG, H.; AHLSTRAND, B.; LAMPEL, J.. Safári de Estratégia: um roteiro pela selva do planejamento estratégico. 2 ed. Porto Alegre: Bookman, 2010.

MUZZIO, H.; COSTA, F. J.. Para além da homogeneidade cultural: a cultura organizacional na perspectiva subnacional. Cad. EBAPE. BR, v.10, n.1, p.146-161, 2012.

NOE, R. A.. An investigation of the determinants of successful assigned mentoring relationships. Personnel Psychology, v.41, n.3, p.457-479, 1988.

O'CONNOR, J.; GU, X.. Developing a Creative Cluster in a Postindustrial City: CIDS and Manchester. The Information Society, v.26, p.124-136, 2010.

RAGINS, B. R.; COTTON, J. L.; MILLER, J. S.. Marginal mentoring: the effects of type mentor, quality of relationship, and program design on work and career attitudes. Academy of Management Journal, v.43, n.6, p.1177-1194, 2000.

SALGUES, L. J. V.. Processos de Mentoria nas organizações em Pernambuco, nas visões dos alunos de MBA Executivo da Universidade Federal de Pernambuco. Dissertação (Mestrado em Administração) - Universidade Federal de Pernambuco, Recife, 2004.

SANTOS JÚNIOR, L. S.. Mentoria e liderança servidora: em busca de um modelo conceitual integrado. Tese (Doutorado em Administração) - Universidade Federal de Pernambuco, Recife, 2017. 
SANTOS JÚNIOR, L. S.. O fenômeno da mentoria na percepção dos professores do curso de administração da Faculdade Integrada do Recife: insights para um modelo brasileiro de mentoria. Dissertação (Mestrado em Administração) - Universidade Federal de Pernambuco, Recife, 2005.

SERPA, A.. A percepção do espaço em um contexto metropolitano. In: CARLOS, A. F. A.; SOUZA, M. L.; SPOSITO, M. E. B.. A Produção do Espaço Urbano: Agentes e Processos, Escalas e Desafios. São Paulo: Contexto, 2013. p.97-108.

TYLER, K.. Mentoring programs link employees and experienced execs. HRMaganize, 1998.

VAN DE VEN, A. H.; POOLE, M. S.. Explaining Development and Change in Organizations. Academy of Management Review, v.20, n.3, p.510-540, 1995.
VIATOR, R. E.. An analysis of formal mentoring programs and perceived barriers to obtaining a mentor at large public accounting firms. Accounting Horizons, v.13, n.1, p.37-53, 1999.

VINODRAI, T.. Design in a downturn? Creative work, labour market dynamics and institutions in comparative perspective. Journal of Regions, Economy and Society, Cambridge, v.6, p.159-176, 2013.

WERNERFELT, B.. A resource-based view of the firm. Strategic Management Journal, v.5, p.171-180, 1984.

ZEY, M. G.. The mentor connection: strategic alliances in corporate life. Londres: Transaction, 1984.

A CBPC - Companhia Brasileira de Produção Científica (CNPJ: 11.221.422/0001-03) detém os direitos materiais desta publicação. Os direitos referem-se à publicação do trabalho em qualquer parte do mundo, incluindo os direitos às renovações, expansões e disseminações da contribuição, bem como outros direitos subsidiários. Todos os trabalhos publicados eletronicamente poderão posteriormente ser publicados em coletâneas impressas sob coordenação da Sustenere Publishing, da Companhia Brasileira de Produção Científica e seus parceiros autorizados. Os (as) autores (as) preservam os direitos autorais, mas não têm permissão para a publicação da contribuição em outro meio, impresso ou digital, em português ou em tradução. 